\title{
Study of Electrical Properties of Silver Nanoparticles deposited on Porous Silicon
}

\author{
Mohammed Ibrahim Ismael ${ }^{*}$, Ghazwan Ghazi Ali \\ Department of Physics, College of Education for Pure Science, University of Mosul, Mosul, Iraq \\ E-mail: ${ }_{1 *}$ Mohammed.esp122@student.uomosul.edu.iq, 라.ghazwan39@uomosul.edu.iq
}

(Received March 06, 2021; Accepted April 19, 2021; Available online September 01, 2021)

DOI: 10.33899/edusj.2021.129664.1147, (C 2021, College of Education for Pure Science, University of Mosul.

This is an open access article under the CC BY 4.0 license (http://creativecommons.org/licenses/by/4.0/).

\begin{abstract}
ABSTRUCT
In this paper, porous silicon has been prepared and studied by photochemical etching method using a ntype silicon wafer with electrical resistivity (0.01-0.02 $\Omega . \mathrm{cm})$, orientation (100), 20\% HF concentration of hydrofluoric acid, current density of $15 \mathrm{~mA} / \mathrm{cm}^{2}$ and etching time at (5min). Silver nanoparticles (AgNPs) have been deposited using laser ablation by drop casting by different laser energy of 400,600 and $800 \mathrm{~m} \mathrm{~J}$. The electrical properties (I-V measurements) of silver nanoparticles on porous silicon have studied in both light and dark conditions. It can be seen the samples behave a rectifier and the current density increases with increase laser energy due to increase in concentration of silver nanoparticles, which lead to a decrease in the values of resistivity as the laser energy increases, this is attributed to the pores are filled with silver nanoparticles and lead to interference between silver and the porous silicon layer. The silver nanoparticles play an important role in forming a homogeneous layer and enhancing the crystal stability of the porous silicon layer.
\end{abstract}

Keywords: Porous silicon, Silver nanoparticles, Electrical properties.

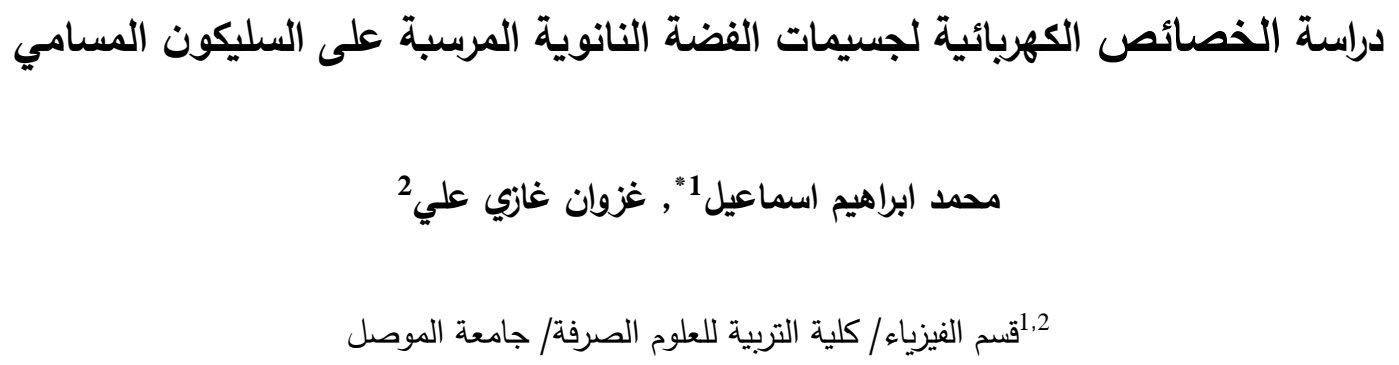

\footnotetext{
الملخص

يهدف البحث إلى تحضير ودراسة خصائص السليكون المسامي بطريقة التتميش الكهروكيميائي الضوئي باستخدام شريحة السليكون نوع n-type ذات مقاومة كهربائية (10.cm 0.02 -0.01) وذات اتجاهية (100) وبتركيز 20 \%

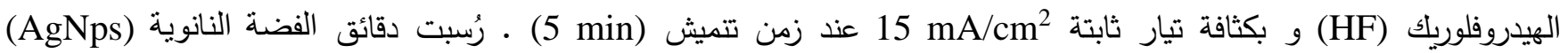
باستخدام الليزر النبضي ذي القدرة العالية (Pulsed Laser ablation) بطريقة التقطير (drop casting) وباستخدام طاقات مختلفة لليزر النبضي (400,600 and 800mJ). بينت نتائج الخصائص لكهربائية (I-V measurements) لجسيمات الفضة النانوية المرسبة على السليكون المسامي ئي حالتي الاضاءة والظلام. بأنَ جميع العينات تسلك سلوكاً تقويمياً (rectifier) وان
} 
كثافة التيار تزداد مع زيادة طاقة الليزر , والسبب يعود في ذلك الى زيادة تركيز جسيمات الفضة النانوية التي تؤدي بدورها الى انخفاض

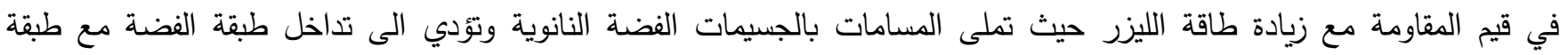
السليكون المسامي • وتؤدي الجسيمات الفضة النانوية دوراً مهماً في تكوين طبقة متجانسة وتعزز الاستقرار البلوري لطبقة لطبة السليكون المسامي.

الكلمات المفتاحية: السليكون المسامي PSi, جسيمات الفضة النانوية AgNPs, الخصائص الكهربائية.

(المقدمة

تعدَ تقنية الاستئصال بالليزر النبضي (pulsed laser ablation ) احدى التقنيات الفيزيائية المستخدمة لتحضير جسيمات نانوية باستعمال تقنية من الاعلى الى الاسفل وذلك باستخدام ليزر ذي طاقة عالية وبنبضات قصيرة مؤثرة على المعدن , تتم تلك

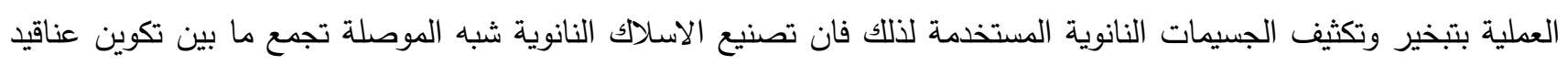
بأحجام نانوية باستخدام الليزر النبضي وبين النمو الحاصل بخار - سائل - صلب (VLS) للمادة المستخدمة ـ تعتبر هذه الطريقة سهلة جداً واقل كلفة كما ان الجسيمات النانوية المتولدة تكون متجانسة نسبيا [1]. يعد السليكون المسامي المؤلف من التراكيب النانوية هو أحد المواد المهمة جداً والتي فتحت أفاقاً واسعة في مجال التطبيقات الكهروضوئية نسبة الى مساحته السطحية الكبيرة

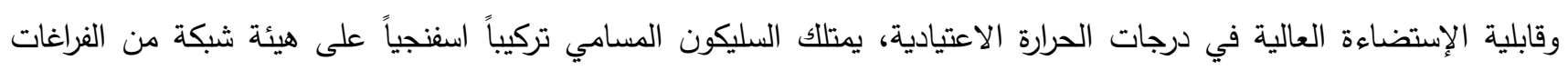

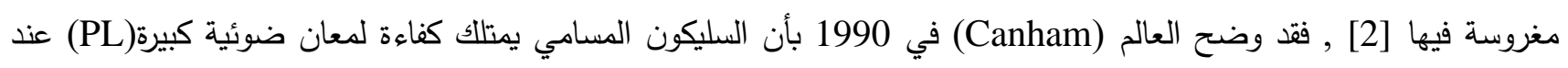

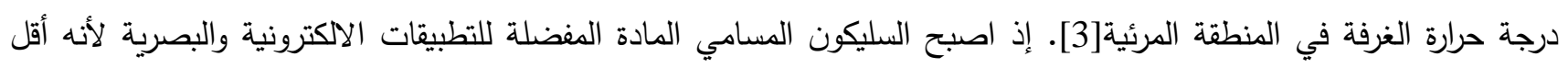
كلفة وأقل أستهلاكا للطاقة. إن الاهتمامات الفعالة في المواد النانوية تتبع من خصائصها الفريدة الفيزيائية والكيميائية والوظيفية التي التي غالباً ما تختلف كثيراً عن نظائرها من المواد الحجمية (Bulk materials) ـ فالعديد من هذه الخصائص الفريدة من نوعها واعدة

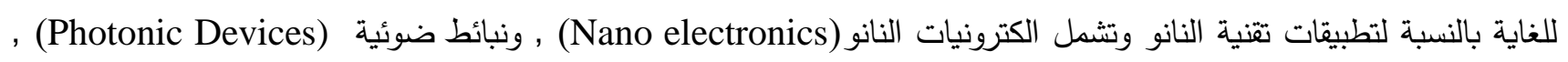

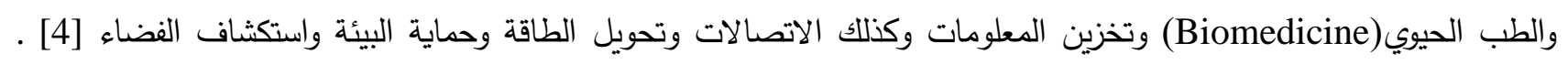
الهدف من هذا البحث هو دراسة الخصائص الكهربائية(B-V measurements) لجسيمات الفضة النانوية المرسبة على السليكون

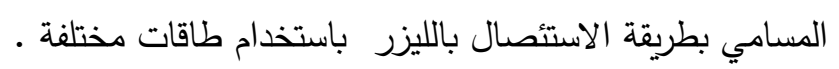

\section{الجزء العملي}

تتكون منظومة التتميش الكهروكيميائي الضوئي من مجز قدرة (DC Power supply) نوع (THAOXIN) يستخدم

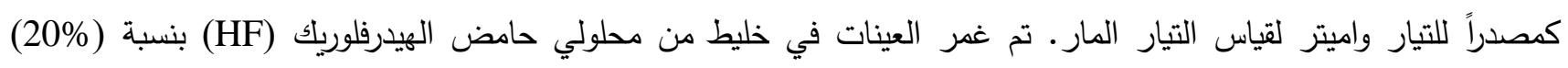
والايثانول(C2 ${ }^{2} \mathrm{H}_{5} \mathrm{OH}$. إذ تم وضع المحلول في خلية مصنوعة من مادة التفلون المقاومة للأحماض القوية كحامض الهيدرفلوريك, ووضع معدن من الفولاذ المقاوم للصدأ في الجزء السفلي من الخلية (Stainless steel) والذي صمم بشكل دقيق لأجل التماس مادة أثناء عملية الأنودة , إذ وضعت شريحة السليكون أسفل القرص الدائري المصنوع من مادة التفلون والذي يحتوي على فتحة دائرية

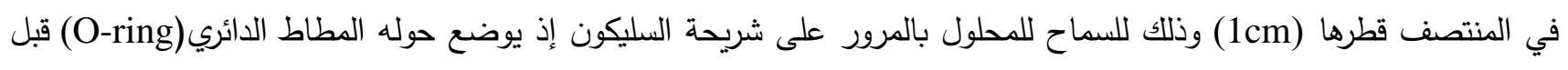

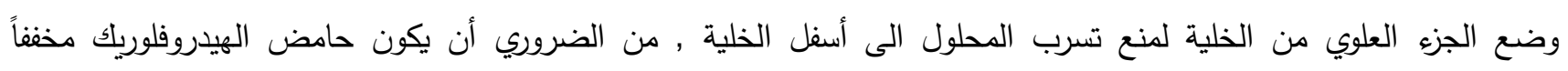
بالإيثانول لإزالة فقاعات الهيدروجين أثثاء عملية التتميش وكذلك الحصول على مسامات متجانسة, تم استخدام قطبين للخلية

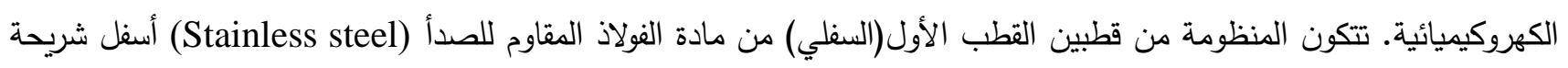
السليكون(الأنود) والقطب الثاني (العلوي) من مادة الذهب(Gold) أعلى شريحة السليكون(الكاثود) , تم استخدام مصدر ضونئي 
تتكستن 100mW/cm²m وكما في الشكل (1), بعد الانتهاء من ربط الدائرة الكهربائية أجريت العملية عند زمن تتميش (5min)

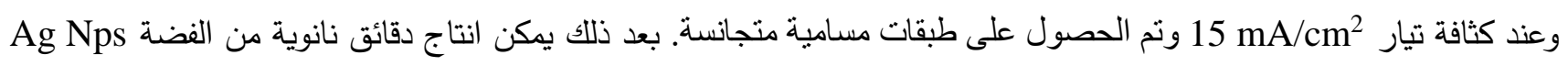
عن طريق الليزر النبضي ذو الطاقة العالية وذلك بغمر معدن الفضة (ذو نقاوة عالية) في وعاء زجاجي موضوع الى الاسفل يحتوي

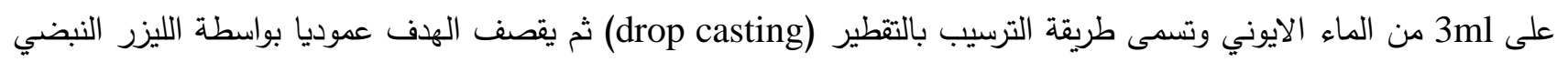
ليزوله الموجي) (1064nm) وبطاقات مختلفة (400,600,800 mJ وعدد نبضات (200) نبضة ولتحقيق اقصى شدة لليزر يستخدم عدسة ذات بعد (12cm) بينها وبين الهدف كما في الثكل (2) الذي يمثل المنظومة العملية لليزر النبضي

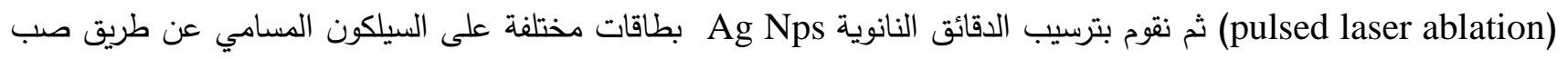

$$
\text { القالب كما في الثكل (3), كما يوضح الثكل (4) صور لمحاليل }
$$
الفضة النانوية المحضرة بطريقة الاستئصال بالليزر .
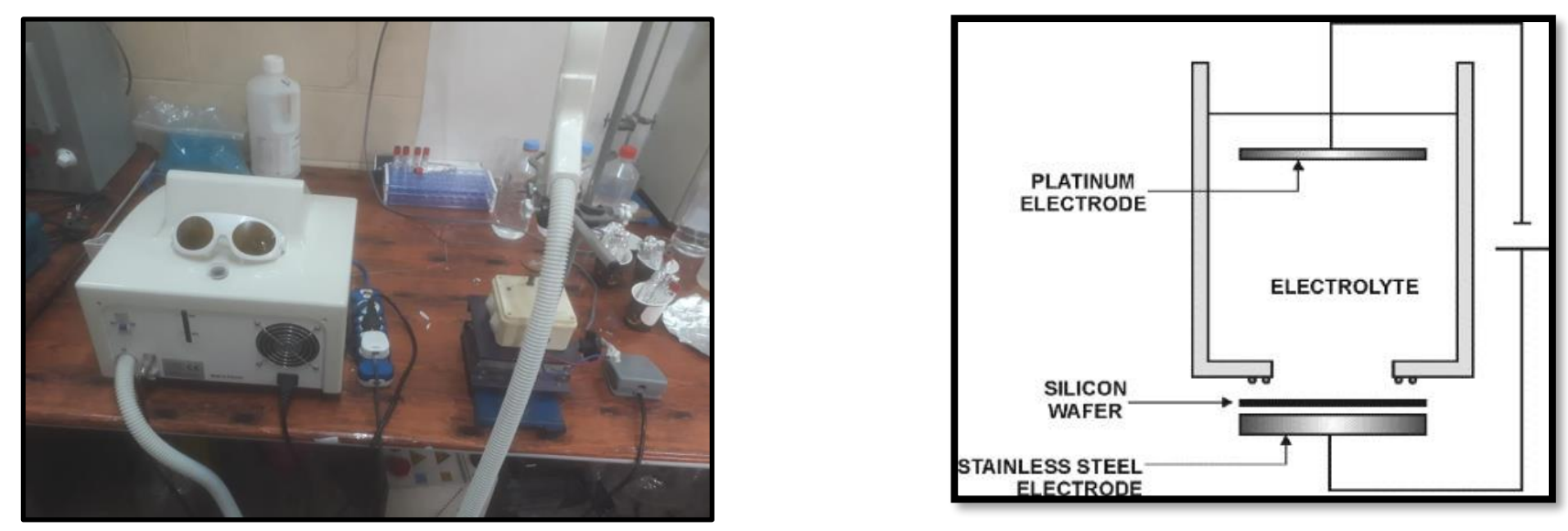

الثكل 2 : المنظومة العملية لليزر النبضي (pulsed laser ablation).

الشكل 1: رسم تخطيطي لخلية الأنودة.

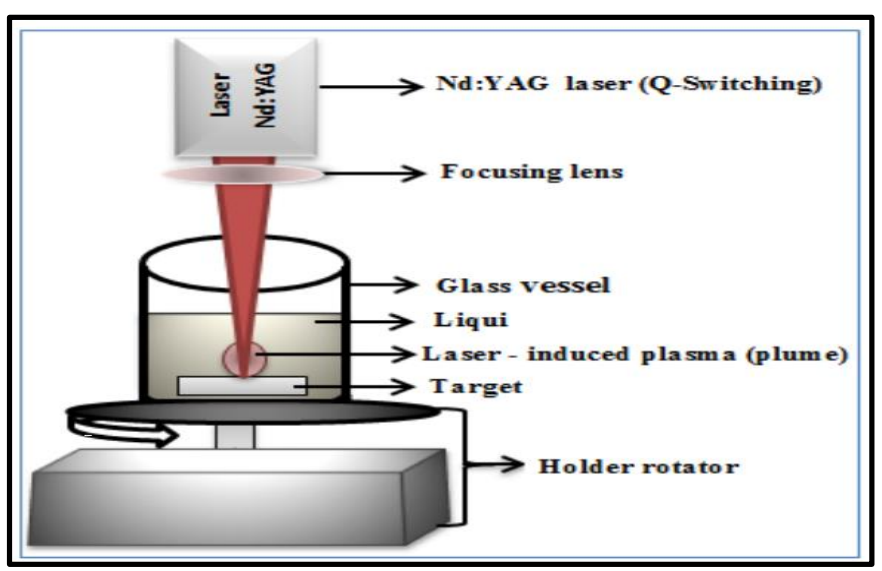

الثكل 3 : عملية تحضير السليكون المسامي وطويقة استخدام الليزر النبضي لترسيب دقائق الفضة النانوية. 

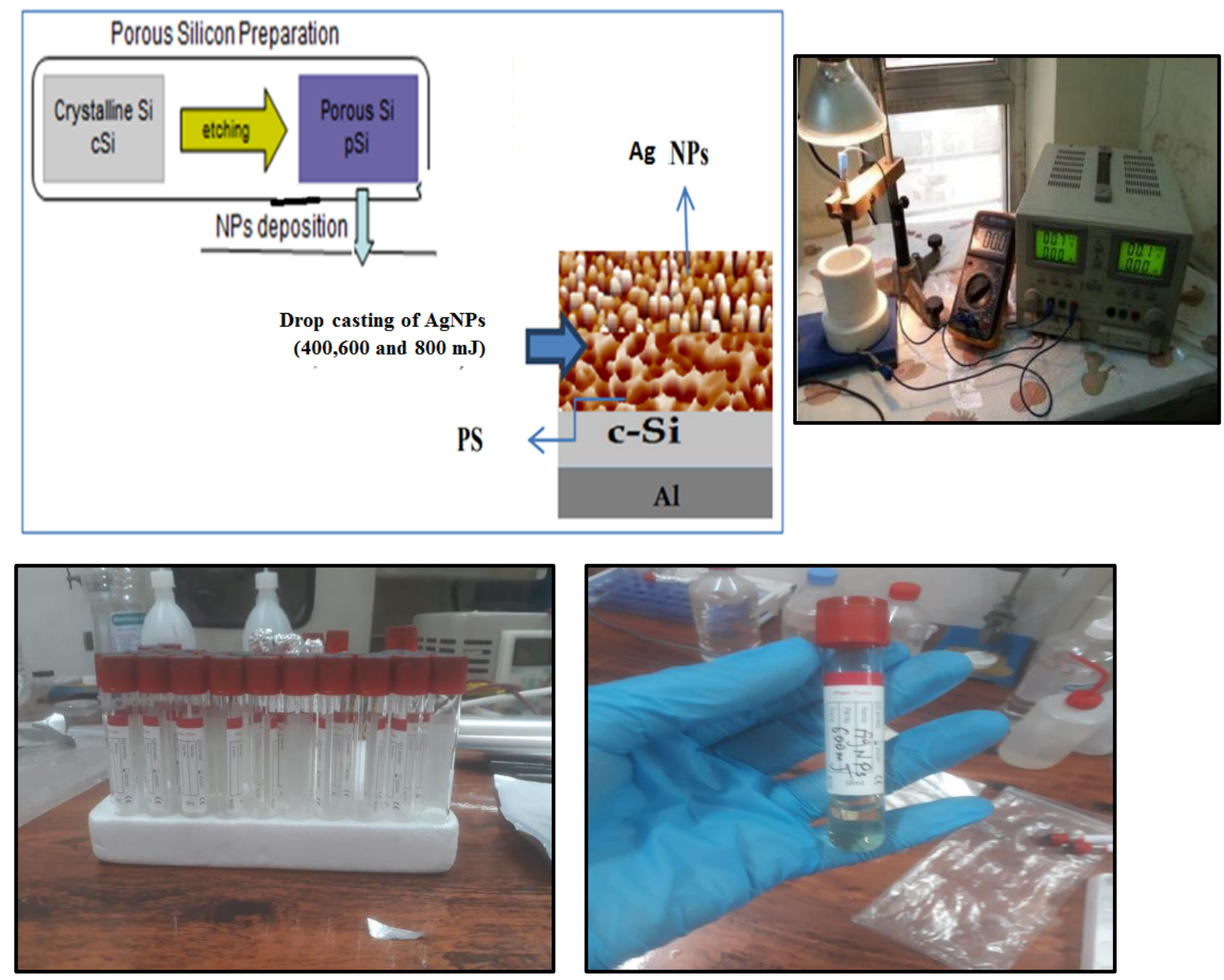

الشكل 4 : محاليل الفضة النانوية المحضرة بطريقة الليزر النبضي (pulsed laser ablation).

\section{النتائج والمناقشة}

خصائص (تيار - فولتية) في الظلام

تم إجراء قياسات الخصائص الكهربائية (J-V) لعينات السليكون المسامي قبل وبعد ترسيب جسيمات الفضة النانوية عند زمن تتميش min 5 للانحياز الأمامي والعكسي في حالة الظلام وتم قياس التيار الخارجي, نلاحظ من الشكل(5) ان خصائص التيار الفولطية تكون ضعيفة للسليكون المسامي , والسبب يعود الى تكوين مراكز اصطياد للحاملات( Capture centers) واعادة الالتحام للتيار الذي نشأ من البنية المسامية للسليكون المسامي, وهذا يؤدي الى زيادة المقاومية ونقصان في مقدار التيار المار عند السطح [5,6]. بعد ترسيب جسيمات الفضة النانوية على سطح السليكون المسامي بطريقة الاستئصال بالليزر النبضي وباستخدام طاقات (400,600 and 800 mJ) , تمت ملاحظة ان خصائص التيار - الفولطية حدث بها انهيار , وزيادة فعالة في قيم كثافة التيار مع زيادة طاقة الليزر مقارنة بالسليكون المسامي , وادى زيادة كثافة التيار الى زيادة في تركيز جسيمات الفضة النانية الثية والتي

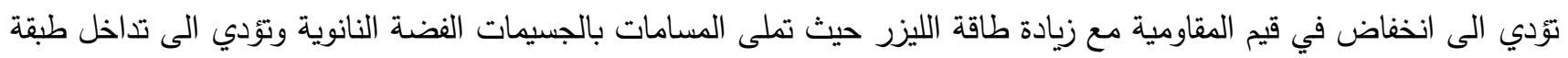
الفضة مع طبقة السليكون المسامي حيث ان قيم المقاومية للسيلكون المسامي كانت 3.cm 3.cm بينما قيم المقاومية 


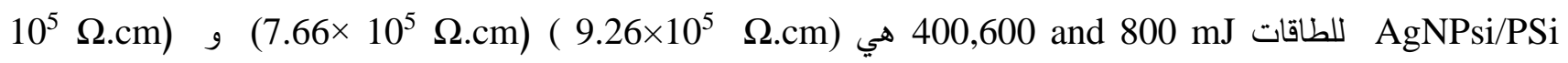
×5.65) على التوالي , معنى ذلك ان العينات تسلك سلوك شوتكي [7] ـ نلاحظ وجود منطقتين في حالة الانحياز الأمامي, منطقة

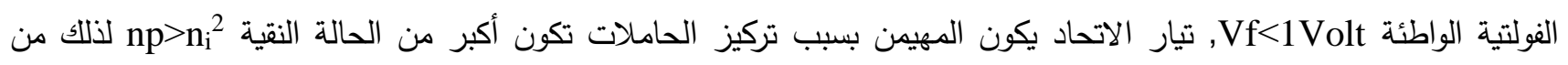

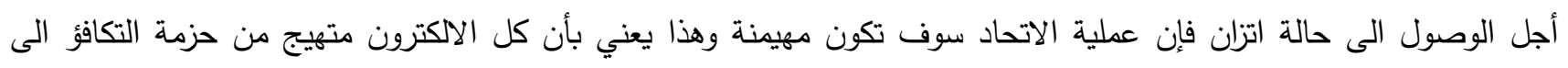

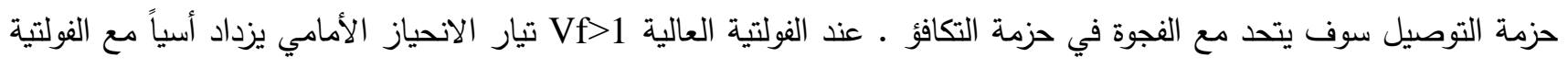

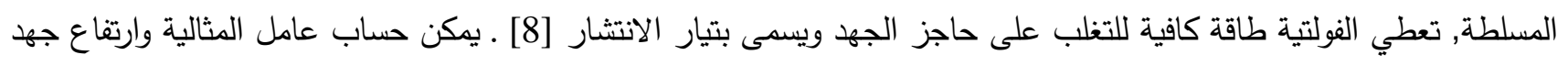

$$
\begin{gathered}
\Phi_{B}=\frac{k_{B} T}{q} \ln \left(\frac{A^{* *} T^{2}}{J}\right) \\
n=\frac{q}{K_{B} T} \frac{\Delta V}{\ln \frac{I}{I_{S}}}
\end{gathered}
$$

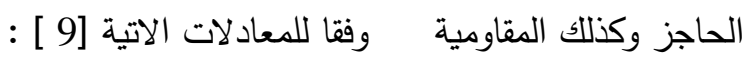

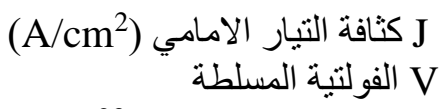

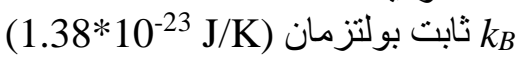
n ثابت ريجاردسون

$$
\boldsymbol{\rho}_{P S i}=\frac{d V}{d J . d p s i}
$$

إذ ان

(J-V) الميل الخطي المستخرج من العلاقة dV/dJ

dpsi

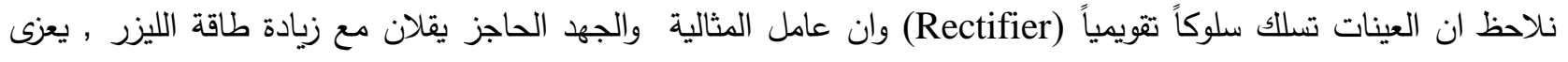
ذللك الى آلية نقل التيار عن طريق إعادة اتحاد حاملات الثحنة [10].

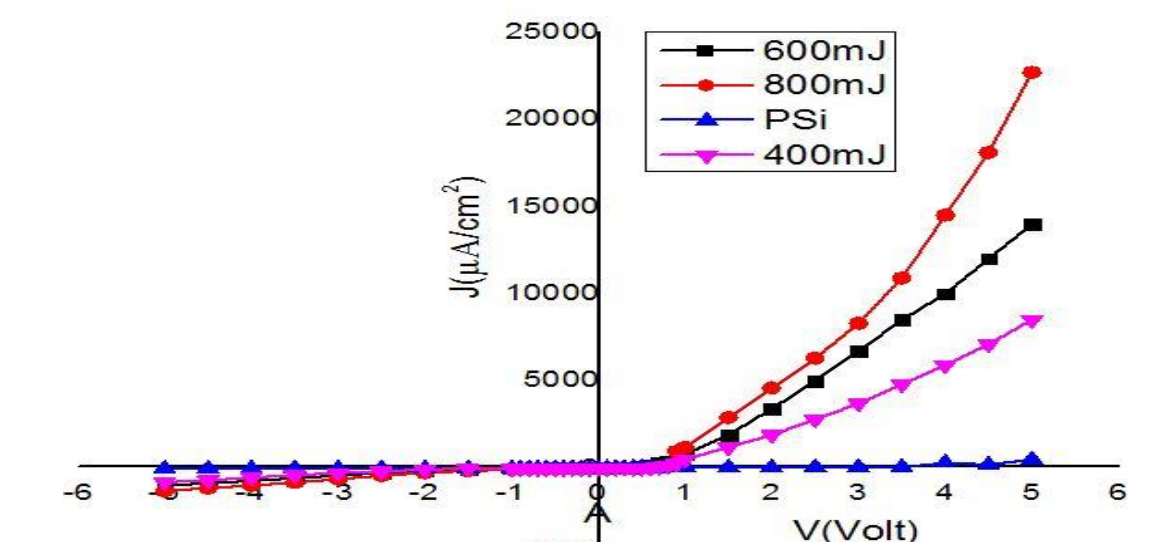

الثكل 5 : قياسات (J-V) في الظلام لعينات السليكون المسامي قبل وبعد ترسيب جسيمات الفضة النانوية عند زمن 5min وعند طاقات 400,600and 800mJ.

خصائص (تيار - فولتية) في الاضاءة

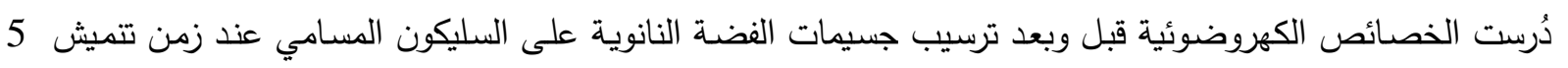

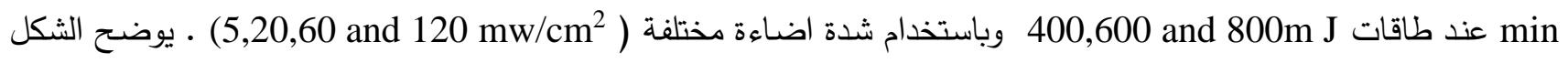

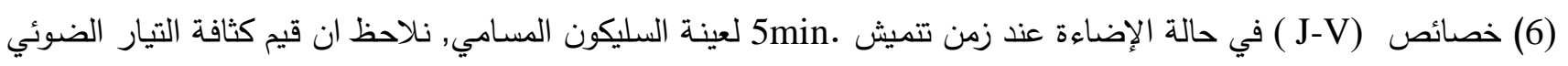


تزداد تدريجيا مع زيادة شدة الاضاءة ويؤدي ذلك الى زيادة في كثافة الحاملات المتولدة عند السطح مما يؤدي بدوره الى زيادة في

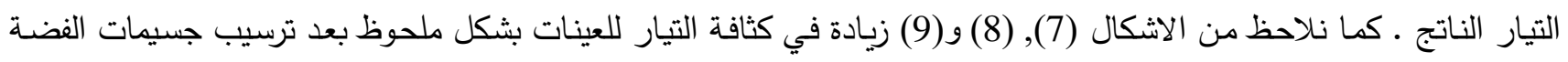
النانوية بسبب زيادة في توليد ازواج الكترون - فجوة بشكل فعال في منطقة الاستتزاف بين طبقات السليكون المسامي وجسيمات الفضة النانوية , يعزي ذلك الى تحسين امتصاص الضوء الناتج عن وجود جزيئات الفضـة النانوية على السليكون المسامي خلال عملية الرنين البلازمون السطحي. ان زيادة في كثافة الحاملات يسبب نقصان في قيم المقاومية وهذا يؤدي الى زيادة في التوصيلية الكهربائية ـ من ناحية اخرى , تؤدي زيادة شدة الضوء الى زيادة التيار الضوئي الذي تم ملاحظته مما يشير الى خصائص فئسية فئية

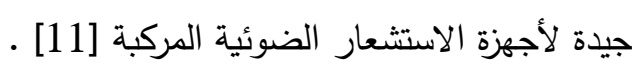

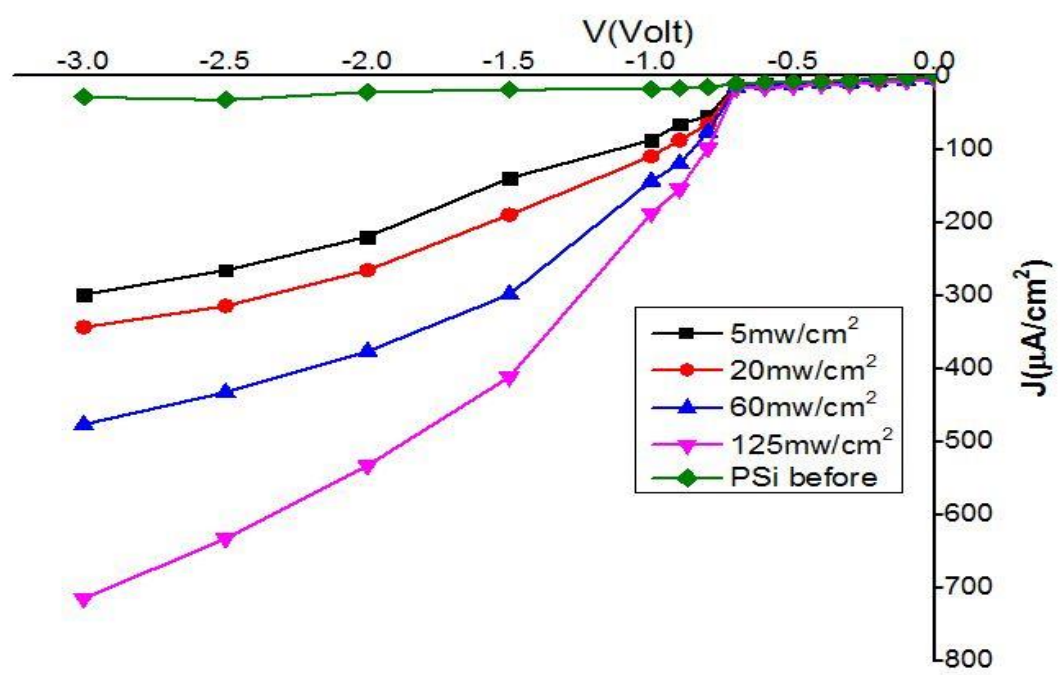

الثكل 6 : قياسات (J-V) في الاضاءة لعينة السليكون المسامي قبل تزسيب جسيمات الفضة النانوية عند زمن 5min وعند شدة اضاءة 5,20,60 and $125 \mathrm{mw} / \mathrm{cm}^{2}$

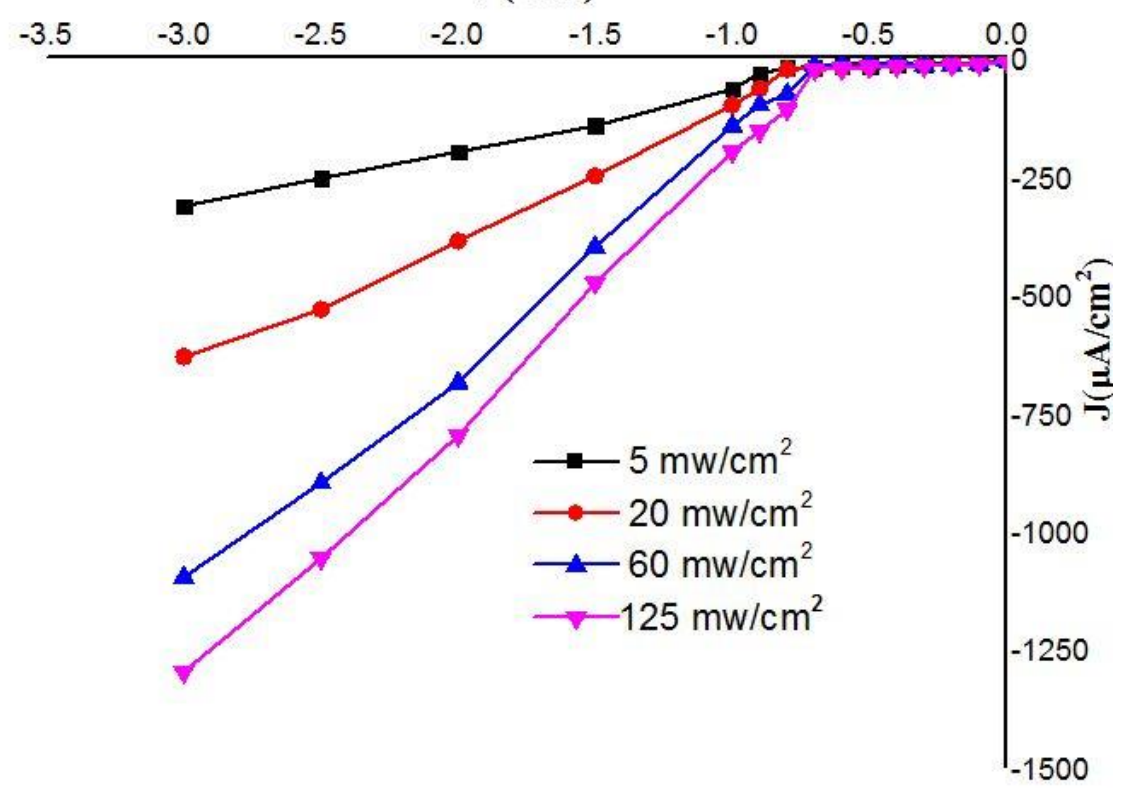

الثكل 7 : قياسات (J-V) في الاضاءة لعينة السليكون المسامي بعد تزسيب جسيمات الفضة النانوية عندزمن

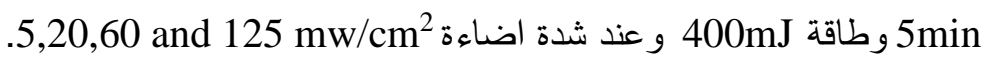




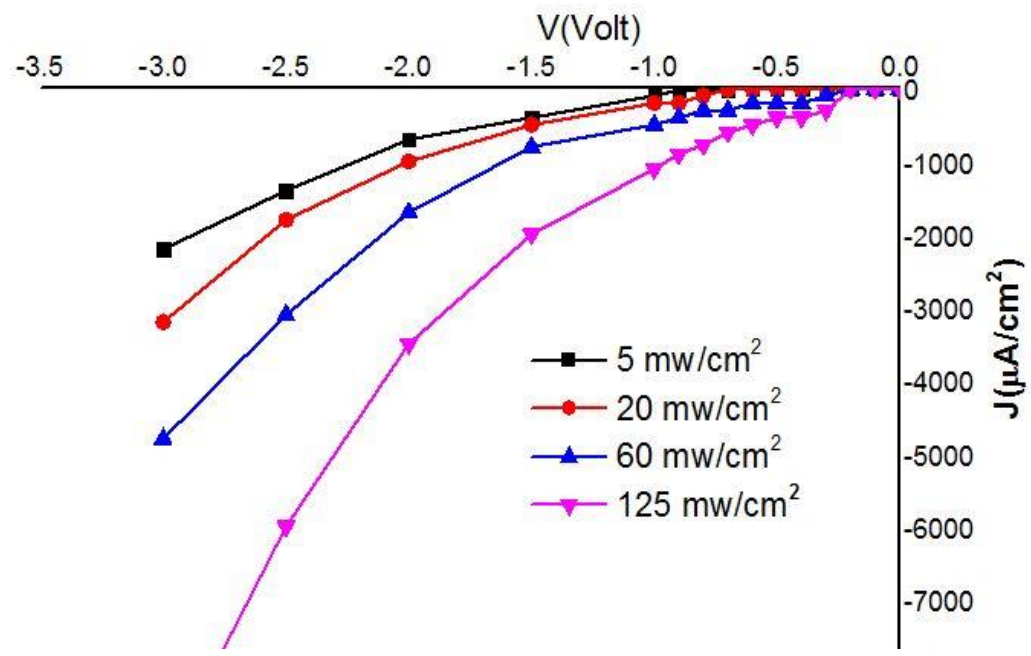

الثكل 8: قياسات (J-V) في الاضاءة لعينة السليكون المسامي بعد ترسيب جسيمات الفضة النانوية عند

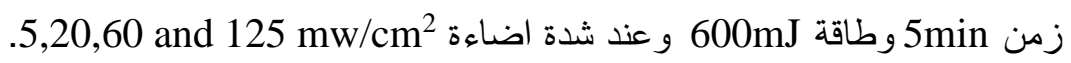

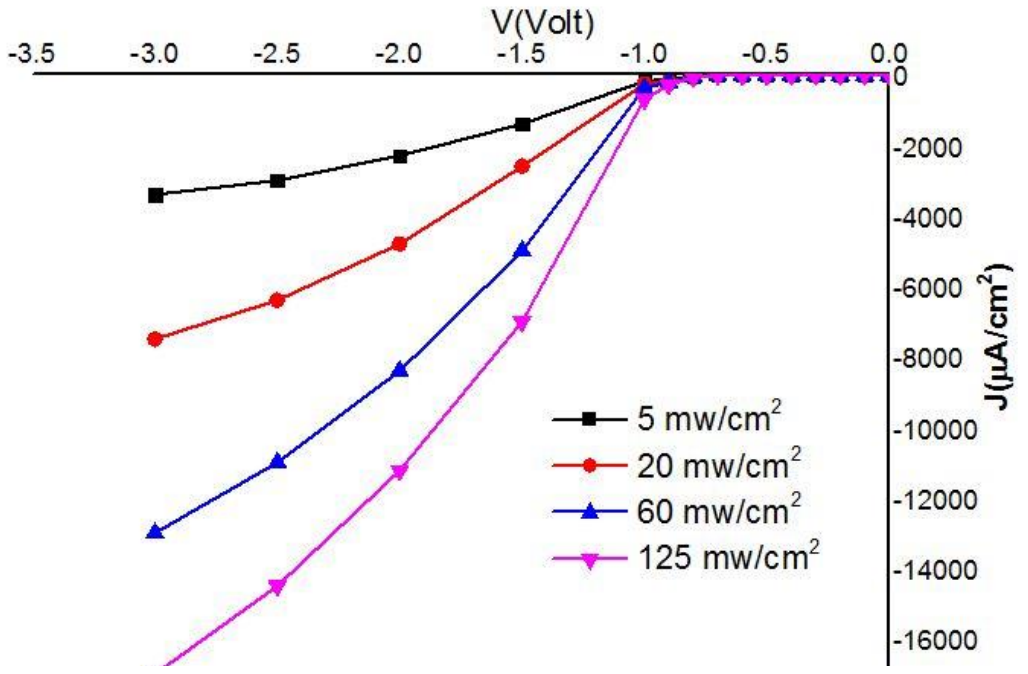

الثكل 9 : قياسات (J-V) في الاضاءة لعينة السليكون المسامي بعد ترسيب جسيمات الفضة النانوية

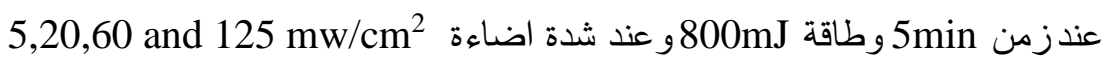

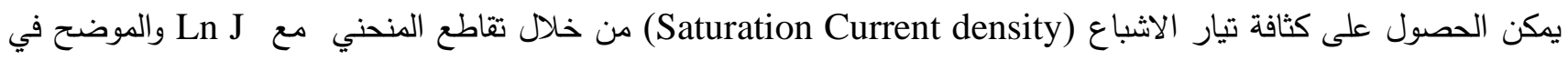
الثكل (10) والذي يبين العلاقة بين Ln J و الفولتية المسلطة (V) , نلاحظ أن كثافة تيار الاشباع تزداد خطيا مع زيادة طاقة

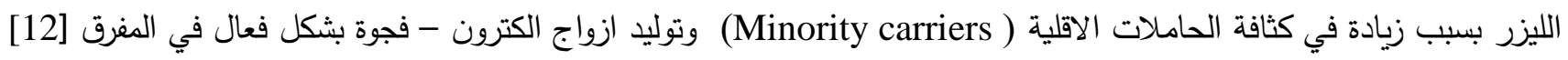

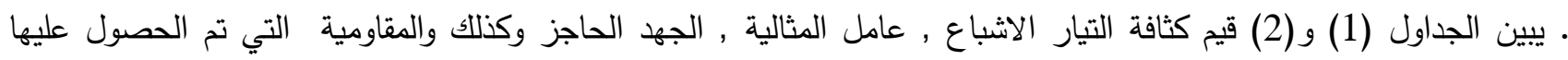

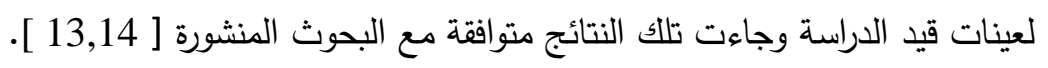




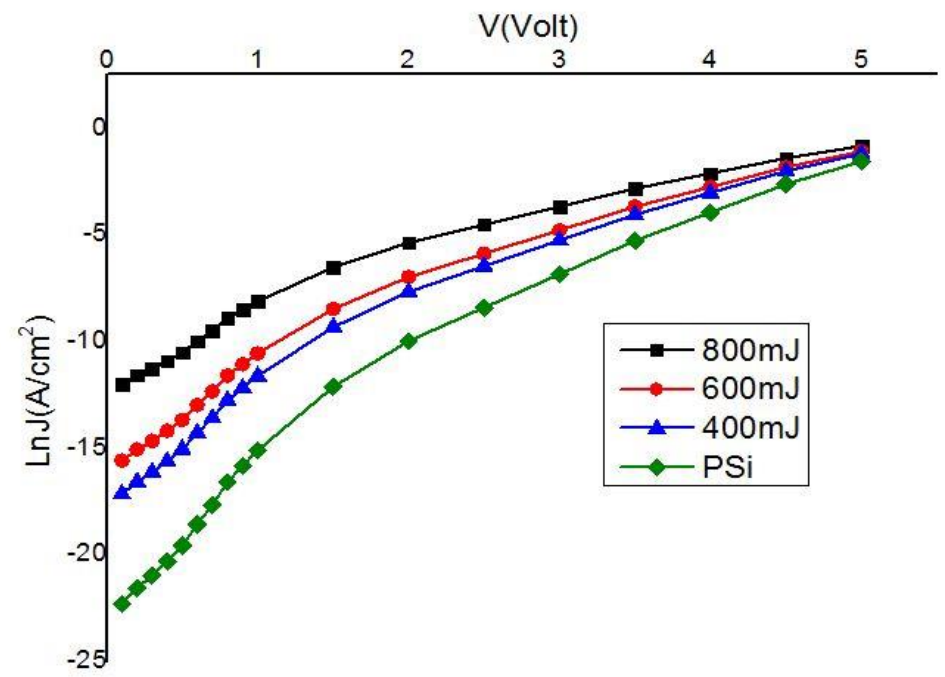

الشكل Ln J مع الفولتية V لعينات السليكون المسامي بعد ترسيب جسيمات الفضة 10 :علاقة النانوية عندزمن 5min ,و عند طاقات 400,600and 800mJ.

جدول1: تيار التشبع العكسي , عامل المثالية , الجهد الحاجز , و المقاومية لعينة السليكون المسامي

\begin{tabular}{ccccc}
\hline $\mathbf{t}(\mathbf{m i n})$. & $\mathbf{J}_{\mathbf{s}}\left(\mathbf{m A} / \mathbf{c m}^{\mathbf{2}}\right)$ & $\mathbf{N}$ & $(\mathbf{e V}) \boldsymbol{\Phi}$ & $\boldsymbol{\rho}(\mathbf{\Omega . c m})$ \\
\hline 5 & 0.0002 & 2.92 & 0.716 & $3.45 \times 10^{6}$ \\
\hline
\end{tabular}

جدول2: تيار التشبع العكسي , عامل المثالية , الجهد الحاجز , و المقاومية لعينات السليكون المسامي بعد الاستئصال بالليزر عند طاقات (400,600 and 800mJ).

\begin{tabular}{ccccc}
\hline $\begin{array}{c}\text { Laser energy } \\
(\mathbf{m J})\end{array}$ & $\mathbf{J}_{\mathbf{s}}\left(\mathbf{m A} \mathbf{/ \mathbf { c m } ^ { 2 }}\right)$ & $\mathbf{N}$ & $(\mathbf{e V}) \boldsymbol{\Phi}$ & $\boldsymbol{\rho}(\mathbf{\Omega . c m})$ \\
\hline 400 & & & & \\
600 & 3.52 & 2.75 & 0.64 & $9.26 \times 10^{5}$ \\
800 & 15.43 & 2.71 & 0.621 & $766 \times 10^{5}$ \\
\hline
\end{tabular}

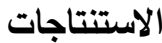

إن النتائج المستحصل عليها في هذا البحث تضيف رؤيا جديدة في مجال تحضير السليكون المسامي وبخصائص جديدة. تم في هذا البحث دراسة تفصيلية لجسيمات الفضة النانوية المرسبة على السليكون المسامي بطريقة الاستئصال بالليزر النبضي (aser (ablation

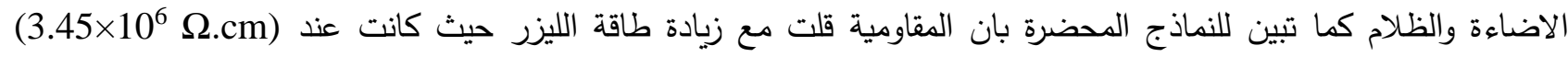

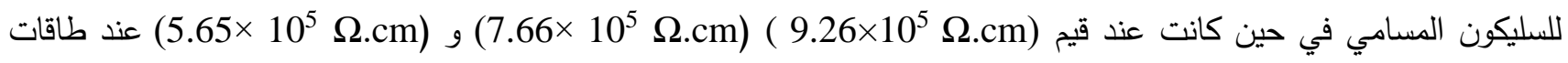
800mJ وكذلك التوالي فضلاً عن ذلك فان التراكيب النانوية للفضة تحسن من اداء السليكون المسامي. شكر وتقدير نود أن نعبر عن شكرنا وتقديرنا لكلية التربية للعلوم الصرفة / قسم الفيزياء في جامعة الموصل على التسهيلات التي كانت مفيدة للغاية في جودة عملنا. 
المصادر

[1] Sharon, E . J. , Nova Sci. publications INC.Newyork 7,9: 98-110 (2011).

[2] Gelloz, B.,Mentek, R. and Koshlda, N.,J. Sol .Sci. and Tech. 3,5 :83-88(2014).

[3] Basu, S. and Kaungo, A., Electro. and Teleco. Engin.66,34: 48-65 (2011).

[4] Hans, J.F. and Werner, M. , Wiley-VCH Verl. GmbH and Co. KGaA, Weinheim 72,45 (2003).

[5] Khashan, K.S., Amany, A. and Mayasaa A., J. Tech. ,27,4:663-674(2008).

[6] Rafid Z., Ban K., Alauldeen S. and Haitham T. H., J. of Res. Lepid. 50,3:82-95(2019).

[7] Raid, A. I., Nahida, J. A. and Rand H.,S., J. Appl. .Nano, 7:109-116(2017).

[8] Maithm, A. O., Suha, A. F. and Ahmed , N . A. , Appl. Sol. state tech., 63, 5 (2020) .

[9] Williams, R. and Rhoderick, E.H., Claredon Press, Oxford ,England (1988).

[10] Kulathuraan, K., Mohanraj, K. and Natarajan, B. , J. Spec. , 152:51-57(2015).

[11] Ahmed, N.A., Nadir, F. H. , Ali H. R. and Hazim , L., J. Nano and Mater. , 11,3:241248(2018).

[12] Warood K. , Luma H., Mohanad Gh. J. of South. Jiao., 54,5: 344-356(2019).

[13] Algun,G. , Arkian, M. , Turk.J.Phys.,23,5 : 789-797(1999).

[14] Naderi, N. and Hashim, M., Inter. J. Electrochem.Sci.,7,4 : 11512-11518 (2012). 